\title{
Long-Term Impact of an Educational Antimicrobial Stewardship Program on Management of Patients with Hematological Diseases
}

\author{
Ana Belén Guisado-Gil 1,2®, Manuela Aguilar-Guisado ${ }^{1, *}$, Germán Peñalva ${ }^{1}$, José Antonio Lepe ${ }^{1}$, \\ Ildefonso Espigado ${ }^{3}{ }^{(0)}$, Eduardo Rodríguez-Arboli ${ }^{3}$, José González-Campos ${ }^{3}$, Nancy Rodríguez-Torres ${ }^{3}$, \\ María Isabel Montero-Cuadrado ${ }^{3}$, José Francisco Falantes-González ${ }^{3}$, Juan Luis Reguera-Ortega ${ }^{3}$, \\ María Victoria Gil-Navarro ${ }^{2}$, José Molina ${ }^{1}$ D, José-Antonio Pérez-Simón $^{3}$ and José Miguel Cisneros ${ }^{1}$ D
}

check for

updates

Citation: Guisado-Gil, A.B.;

Aguilar-Guisado, M.; Peñalva, G.;

Lepe, J.A.; Espigado, I.

Rodríguez-Arbolí, E.;

González-Campos, J.;

Rodríguez-Torres, N.;

Montero-Cuadrado, M.I.;

Falantes-González, J.F.; et al.

Long-Term Impact of an Educational Antimicrobial Stewardship Program on Management of Patients with Hematological Diseases. Antibiotics 2021, 10, 136. https://doi.org/ 10.3390/antibiotics10020136

Academic Editors: Albert Figueras and Diane Ashiru-Oredope

Received: 16 December 2020

Accepted: 27 January 2021

Published: 30 January 2021

Publisher's Note: MDPI stays neutral with regard to jurisdictional claims in published maps and institutional affiliations.

Copyright: (c) 2021 by the authors. Licensee MDPI, Basel, Switzerland. This article is an open access article distributed under the terms and conditions of the Creative Commons Attribution (CC BY) license (https:/ / creativecommons.org/licenses/by/ $4.0 /)$.
1 Department of Infectious Diseases, Microbiology and Preventive Medicine, Infectious Diseases Research Group, Institute of Biomedicine of Seville (IBiS), University of Seville/CSIC/University Hospital Virgen del Rocio, 41013 Seville, Spain; anaguigil@gmail.com (A.B.G.-G.); german.penalva@gmail.com (G.P.); josea.lepe.sspa@juntadeandalucia.es (J.A.L.); josemolinagb@gmail.com (J.M.); jmcisnerosh@gmail.com (J.M.C.)

2 Department of Pharmacy, University Hospital Virgen del Rocio, 41013 Seville, Spain; mariav.gil.sspa@juntadeandalucia.es

3 Department of Hematology, Institute of Biomedicine of Seville (IBiS/CSIC/CIBERONC), University Hospital Virgen del Rocio, University of Seville, 41013 Seville, Spain; ildefonso.espigado.sspa@juntadeandalucia.es (I.E.); edurodarb@gmail.com (E.R.-A.); jose.gonzalez.sspa@juntadeandalucia.es (J.G.-C.); nanarotor@hotmail.com (N.R.-T.); mariai.montero.sspa@juntadeandalucia.es (M.I.M.-C.); josef.falantes.sspa@juntadeandalucia.es (J.F.F.-G.); juanlu_jlr@hotmail.com (J.L.R.-O.); josea.perez.simon.sspa@juntadeandalucia.es (J.-A.P.-S.)

* Correspondence: maguilarguisado@yahoo.es; Tel.: +34-670943816

\begin{abstract}
Antimicrobial stewardship programs (ASPs) in hematological patients are especially relevant. However, information about ASPs in this population is scarce. For 11 years, we quarterly assessed antimicrobial consumption and incidence and death rates of multidrug-resistant (MDR) bloodstream infections (BSI) in the hematology Department. Healthcare activity indicators were also monitored yearly. We performed an interrupted time-series analysis. Antimicrobials showed a sustained reduction with a relative effect of $-62.3 \%(95 \%$ CI -84.5 to -40.1$)$ nine years after the inception of the ASP, being especially relevant for antifungals (relative effect $-80.4 \%,-90.9$ to -69.9 ), quinolones (relative effect $-85.0 \%,-102.0$ to -68.1 ), and carbapenems (relative effect $-68.8 \%,-126.0$ to -10.6 ). Incidence density of MDR BSI remained low and stable (mean $1.10 \mathrm{vs.}$ 0.82 episodes per 1000 occupied bed days for the pre-intervention and the ASP period, respectively) with a quarterly percentage of change of $-0.3 \%(95 \% \mathrm{CI}-2.0$ to 1.4$)$. Early and late mortality of MDR BSI presented a steady trend (quarterly percentage of change $-0.7 \%, 95 \%$ CI -1.7 to 0.3 and $-0.6 \%, 95 \% \mathrm{CI}-1.5$ to 0.3 , respectively). Volume and complexity of healthcare activity increased over the years. The ASP effectively achieved long-term reductions in antimicrobial consumption and improvements in the prescription profile, without increasing the mortality of MDR BSI.
\end{abstract}

Keywords: antimicrobial stewardship; anti-infective agents; bacteremia; candidemia; hematologic diseases

\section{Introduction}

Antimicrobial stewardship programs (ASPs) have been identified as a valuable tool to optimize the antimicrobial use in healthcare centers, improving patient outcomes and reducing adverse events and the selection pressure related to the use of antimicrobial agents [1].

In hematological patients receiving immunosuppressive therapy, collateral damages of antimicrobial consumption, especially broad-spectrum antibiotic therapy, include the selection of multidrug-resistant (MDR) microorganisms [2], an increased propensity to 
fungal infections [3], and microbiota dysbiosis [4]. Although, due to these reasons the impact of ASPs in patients with hematological diseases might be especially relevant, information regarding the development of antimicrobial stewardship strategies in these patients is scarce [5-7].

An ASP named Institutional Program for the Optimization of Antimicrobial Treatment (PRIOAM) started in our institution in January 2011. Since then, assessments of antimicrobial use, in-hospital bacterial resistance, and mortality rates associated with nosocomial bloodstream infections (BSI) have decreased significantly [8,9]. This program covers the entire hospital and presents specific interventions focused on hematological patients.

We hypothesized that a comprehensive ASP in hematological patients could also optimize antimicrobial use, reducing the overall consumption and improving the prescription profile without increasing the incidence and mortality rates of BSI produced by MDR microorganisms. Thus, the objective of the present study was to assess the impact of the PRIOAM on antimicrobial consumption and the incidence and death rates caused by MDR BSI in hospitalized adult patients with hematological diseases.

\section{Results}

Since the inception of the ASP and as part of PRIOAM educative measures (see "Intervention" at the Materials and Methods section), a total number of 218 face-to-face structured educational interviews (EI) were performed (mean $24 \pm 19$ EI per year). The main reasons for inappropriate antimicrobial therapy were: an incorrect selection of the drug according to the suspected diagnosis $(28.4 \%)$ or inappropriate duration $(28.0 \%)$ in the case of empiric treatments, and failing to de-escalate (11.0\%) in the case of targeted therapies.

Moreover, 18 clinical sessions (two per year) were performed about practical aspects of common infections in hematological malignancy patients and 45 reports were produced, including one per quarter and an additional annual report to the head of the department, on the level of attainment of pre-agreed objectives.

\subsection{Antimicrobial Consumption}

The mean consumption of all antimicrobials decreased from $148.2 \pm 16.2$ defined daily doses (DDD) per 100 occupied bed days (OBD) in the pre-intervention period to $112.0 \pm 21.7$ DDD per 100 OBD in the ASP period $(p<0.001)$. Detailed data from the pre-post analysis are included in the Supplementary material (Table S1).

The interrupted time-series (ITS) analysis (Table 1, Figures 1-3) showed a sustained reduction in favor of the intervention with a relative effect of $-62.3 \%(95 \%$ confidence interval $[\mathrm{CI}]-84.5$ to -40.1 ) nine years after the inception of the ASP, when compared with the expected antimicrobial consumption based on the pre-intervention trend. As for antibiotics, a prompt change in the level after the inception of the ASP of -17.22 DDD per 100 OBD (95\% CI -29.17 to -5.28$)$ was found. Regarding antifungal consumption, a decreasing trend with a change in slope of $-3.32 \mathrm{DDD}$ per $100 \mathrm{OBD}(95 \% \mathrm{CI}-6.04$ to -0.60$)$ and a relative effect $-80.4 \%$ (95\% CI -90.9 to -69.9$)$ was obtained with the intervention. Quinolones were the agents that showed the highest reduction with a change in the level of $-18.45 \mathrm{DDD}$ per $100 \mathrm{OBD}(95 \% \mathrm{CI}-25.29$ to -11.62$)$ after the start of the intervention that led to a relative effect of $-85.0 \%(95 \% \mathrm{CI}-102.0$ to -68.1$)$ at the end of the study period. Broad-spectrum antibiotics such as carbapenems and glycopeptides presented significant relative effects of $-68.8 \%(95 \% \mathrm{CI}-126.0$ to -10.6$)$ and $-70.5 \%(95 \% \mathrm{CI}-138.9$ to -2.1$)$, respectively, compared with the expected consumption based on the pre-intervention trend. The global trend is described in Table S2. 
Table 1. Interrupted time-series analysis of changes in trends of antimicrobial consumption.

\begin{tabular}{|c|c|c|c|c|}
\hline Outcomes & $\begin{array}{c}\text { Pre-Intervention } \\
\text { Trend }\end{array}$ & $\begin{array}{l}\text { Change } \\
\text { in Level }^{\text {a }}\end{array}$ & $\begin{array}{l}\text { Change } \\
\text { in Trend } b\end{array}$ & $\begin{array}{l}\text { Relative } \\
\text { Effect }^{\text {c } \%}\end{array}$ \\
\hline Total J01+J02 & $\begin{array}{c}1.83 \\
(-2.14 \text { to } 5.80)\end{array}$ & $\begin{array}{c}-13.98 \\
(-35.65 \text { to } 7.69)\end{array}$ & $\begin{array}{c}-3.52 \\
(-7.57 \text { to } 0.52)\end{array}$ & $\begin{array}{c}-62.3 \\
(-84.5 \text { to }-40.1)\end{array}$ \\
\hline Total antibiotics (J01) & $\begin{array}{c}-0.65 \\
(-2.84 \text { to } 1.54)\end{array}$ & $\begin{array}{c}-17.22 \\
(-29.17 \text { to }-5.28)\end{array}$ & $\begin{array}{c}-0.27 \\
(-2.49 \text { to } 1.95)\end{array}$ & $\begin{array}{c}-32.4 \\
(-99.2 \text { to } 34.5)\end{array}$ \\
\hline Total antifungals (J02) & $\begin{array}{c}2.54 \\
(-0.12 \text { to } 5.20)\end{array}$ & $\begin{array}{c}3.31 \\
(-11.12 \text { to } 17.74)\end{array}$ & $\begin{array}{c}-3.32 \\
(-6.04 \text { to }-0.60)\end{array}$ & $\begin{array}{c}-80.4 \\
(-90.9 \text { to }-69.9)\end{array}$ \\
\hline Carbapenems & $\begin{array}{c}-0.01 \\
(-0.69 \text { to } 0.66)\end{array}$ & $\begin{array}{c}-0.67 \\
(-4.33 \text { to } 2.99)\end{array}$ & $\begin{array}{c}-0.20 \\
(-0.89 \text { to } 0.49)\end{array}$ & $\begin{array}{c}-68.8 \\
(-126.0 \text { to }-10.6)\end{array}$ \\
\hline $\begin{array}{l}\text { Piperacillin- } \\
\text { tazobactam }\end{array}$ & $\begin{array}{c}0.63 \\
(-0.25 \text { to } 1.51)\end{array}$ & $\begin{array}{c}7.78 \\
\text { (3.30 to } 12.27)\end{array}$ & $\begin{array}{c}-0.86 \\
(-1.78 \text { to } 0.07)\end{array}$ & $\begin{array}{c}-67.3 \\
(-96.9 \text { to }-38.6)\end{array}$ \\
\hline $\begin{array}{l}\text { Antipseudomonal } \\
\text { cephalosporins }\end{array}$ & $\begin{array}{c}-0.55 \\
(-1.57 \text { to } 0.47)\end{array}$ & $\begin{array}{c}-10.79 \\
(-16.18 \text { to }-5.41)\end{array}$ & $\begin{array}{c}0.77 \\
(-0.29 \text { to } 1.82)\end{array}$ & $\begin{array}{c}105.1 \\
(-195.6 \text { to } 405.8)\end{array}$ \\
\hline Quinolones & $\begin{array}{c}0.16 \\
(-1.16 \text { to } 1.47)\end{array}$ & $\begin{array}{c}-18.45 \\
(-25.29 \text { to }-11.62)\end{array}$ & $\begin{array}{c}-0.45 \\
(-1.82 \text { to } 0.93)\end{array}$ & $\begin{array}{c}-85.0 \\
(-102.0 \text { to }-68.1)\end{array}$ \\
\hline Amikacin & $\begin{array}{c}-0.03 \\
(-0.46 \text { to } 0.39)\end{array}$ & $\begin{array}{c}1.68 \\
(-0.51 \text { to } 3.87)\end{array}$ & $\begin{array}{c}-0.05 \\
(-0.48 \text { to } 0.39)\end{array}$ & $\begin{array}{c}0.1 \\
(-410.8 \text { to } 413.6)\end{array}$ \\
\hline Glycopeptides & $\begin{array}{c}0.01 \\
(-0.55 \text { to } 0.56)\end{array}$ & $\begin{array}{c}0.68 \\
(-2.27 \text { to } 3.62)\end{array}$ & $\begin{array}{c}-0.17 \\
(-0.74 \text { to } 0.40)\end{array}$ & $\begin{array}{c}-70.5 \\
(-138.9 \text { to }-2.1)\end{array}$ \\
\hline
\end{tabular}

Data are presented as quarterly defined daily doses per 100 occupied bed days with a $95 \%$ confidence interval, unless otherwise specified. ${ }^{a}$ Increase or decrease in the first quarter after the start of the antimicrobial stewardship program (ASP) period with respect to the expected value. ${ }^{b}$ Change in slope for the ASP period. ${ }^{c}$ Percentage difference between the expected value according to the pre-intervention trend and the trend nine years after the start of the ASP.

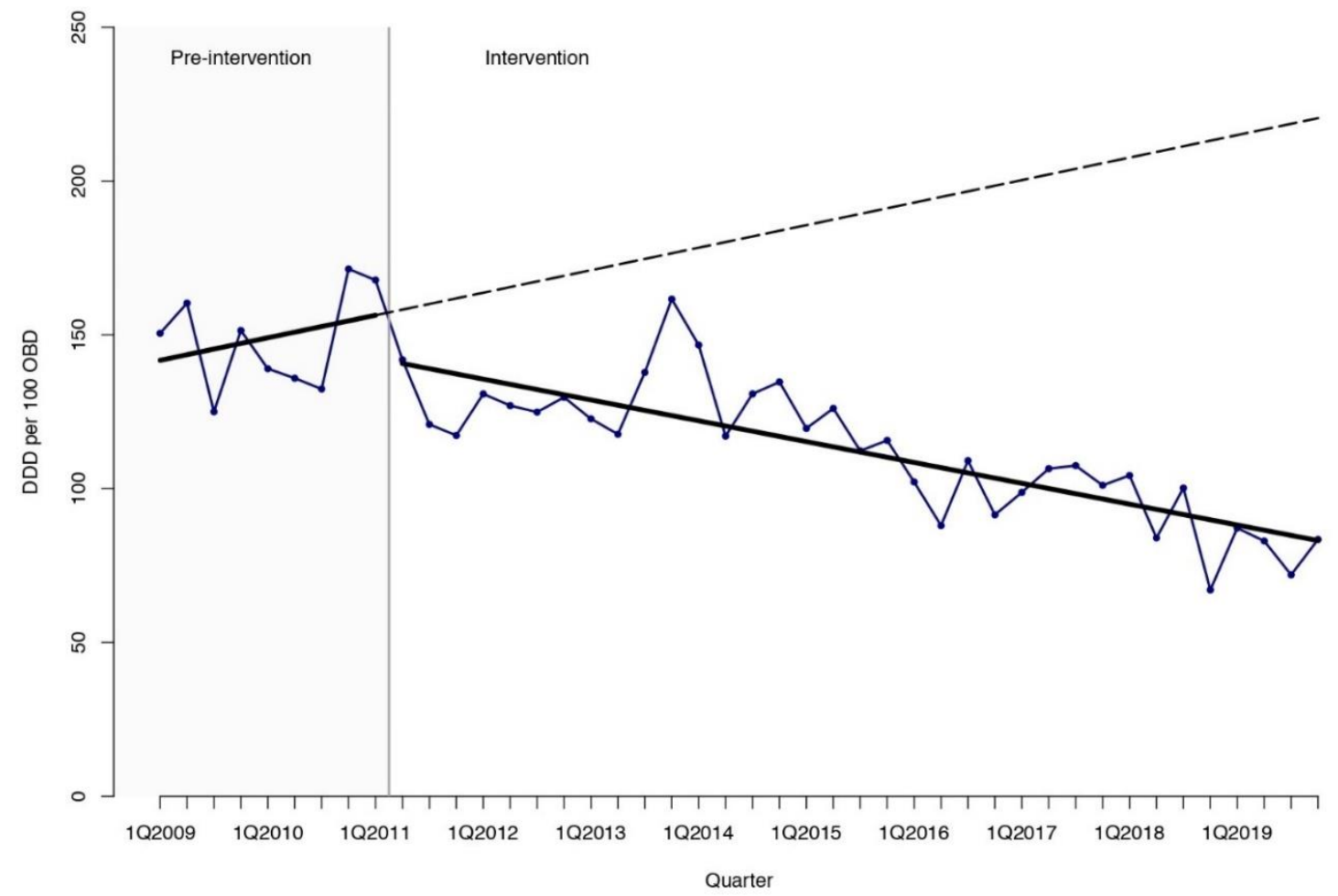

Figure 1. Interrupted time-series analysis of the trends in antimicrobial consumption (antibacterials for systemic use and antifungals) observed before and after the implementation of the antimicrobial stewardship program. Solid lines show the observed trend during the pre-intervention and intervention periods. Dashed lines show the expected trend after the intervention according to the pre-intervention values. DDD, defined daily doses. OBD, occupied bed days. Q, quarter. 
J01

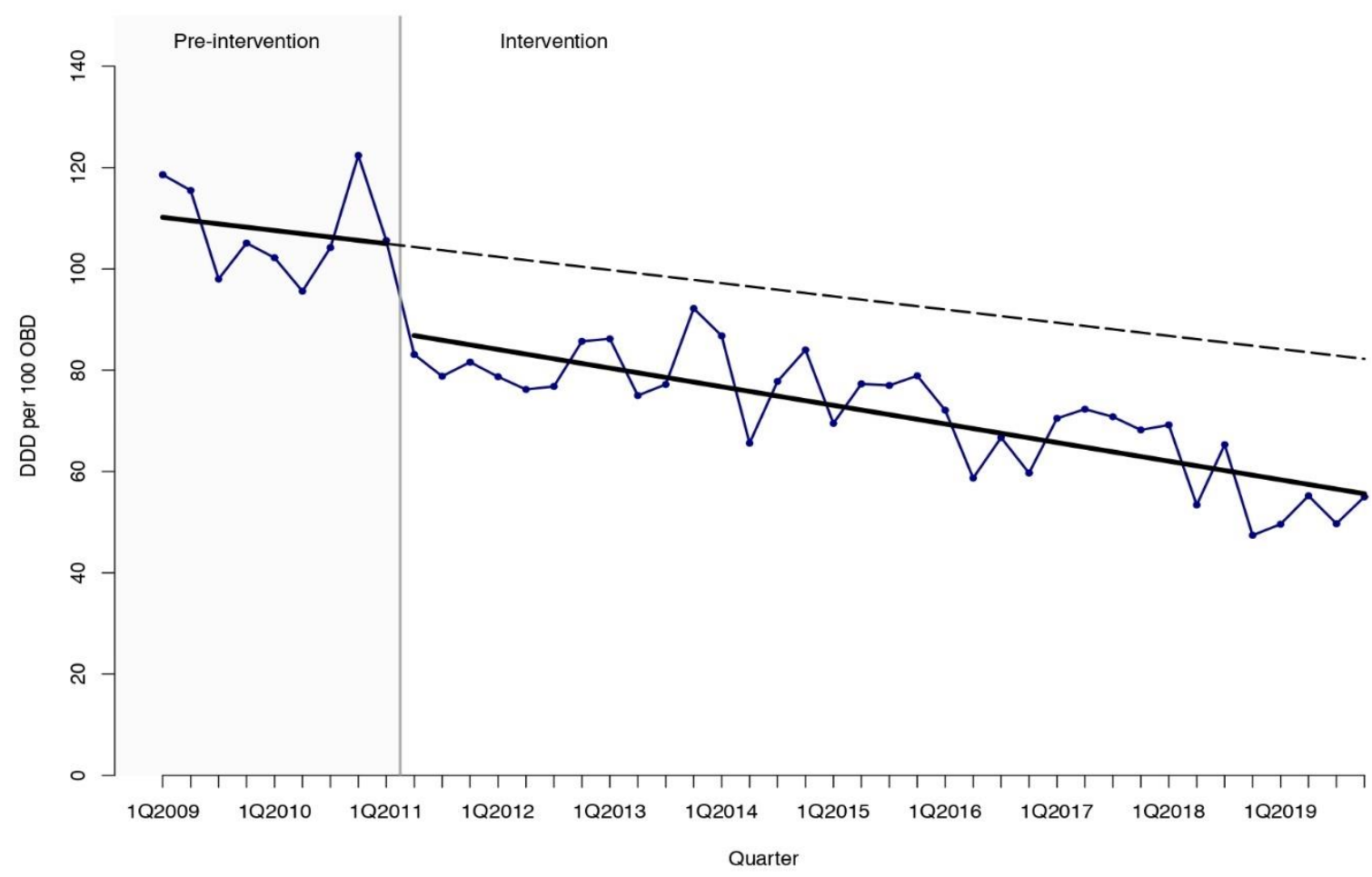

J02

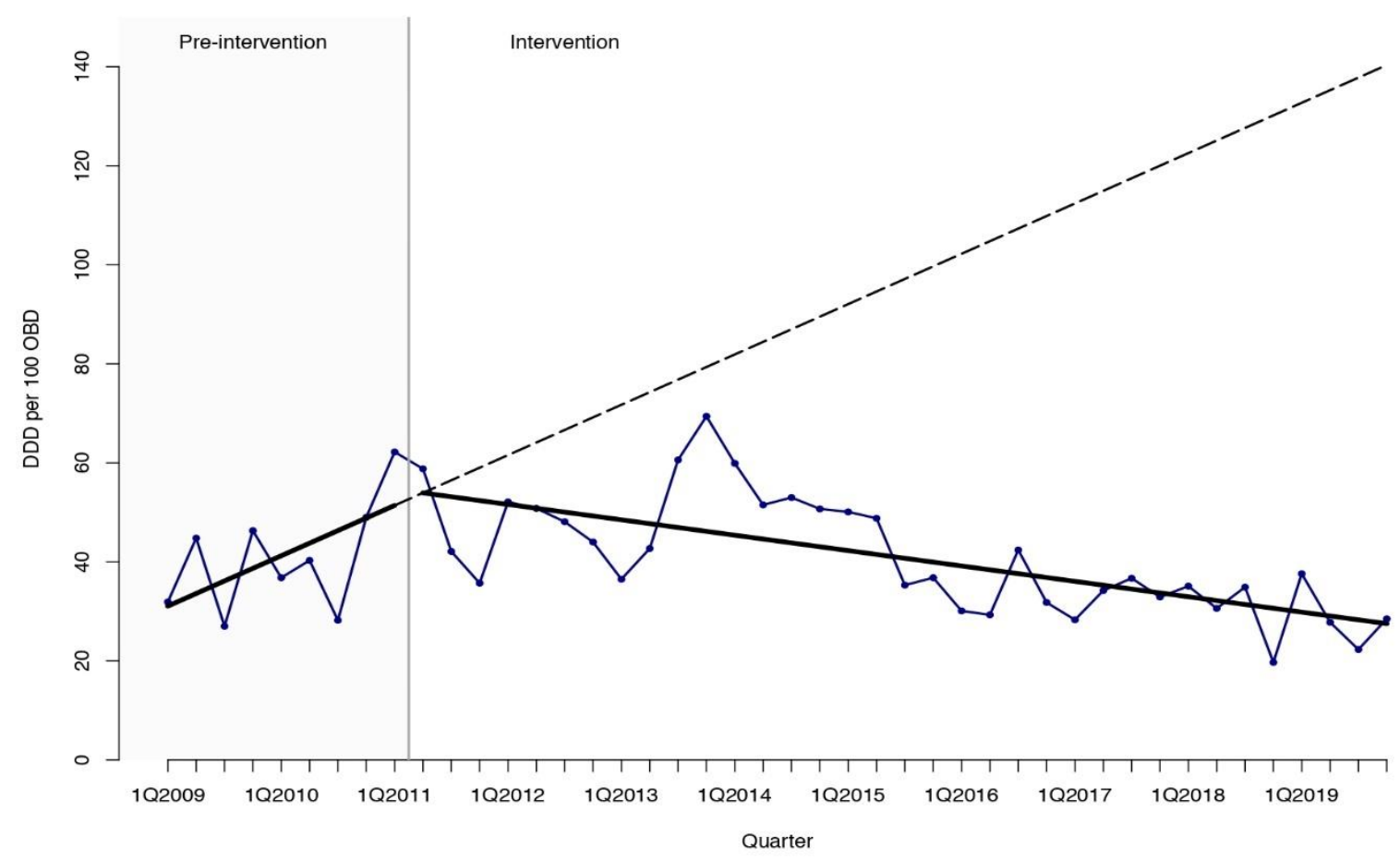

Figure 2. Interrupted time-series analysis of the trends in consumption for antibacterials for systemic use (J01) and antifungals (J02) observed before and after the implementation of the antimicrobial stewardship program. Solid lines show the observed trend during the pre-intervention and intervention periods. Dashed lines show the expected trend after the intervention according to the pre-intervention values. DDD, defined daily doses. OBD, occupied bed days. Q, quarter. 
Carbapenems

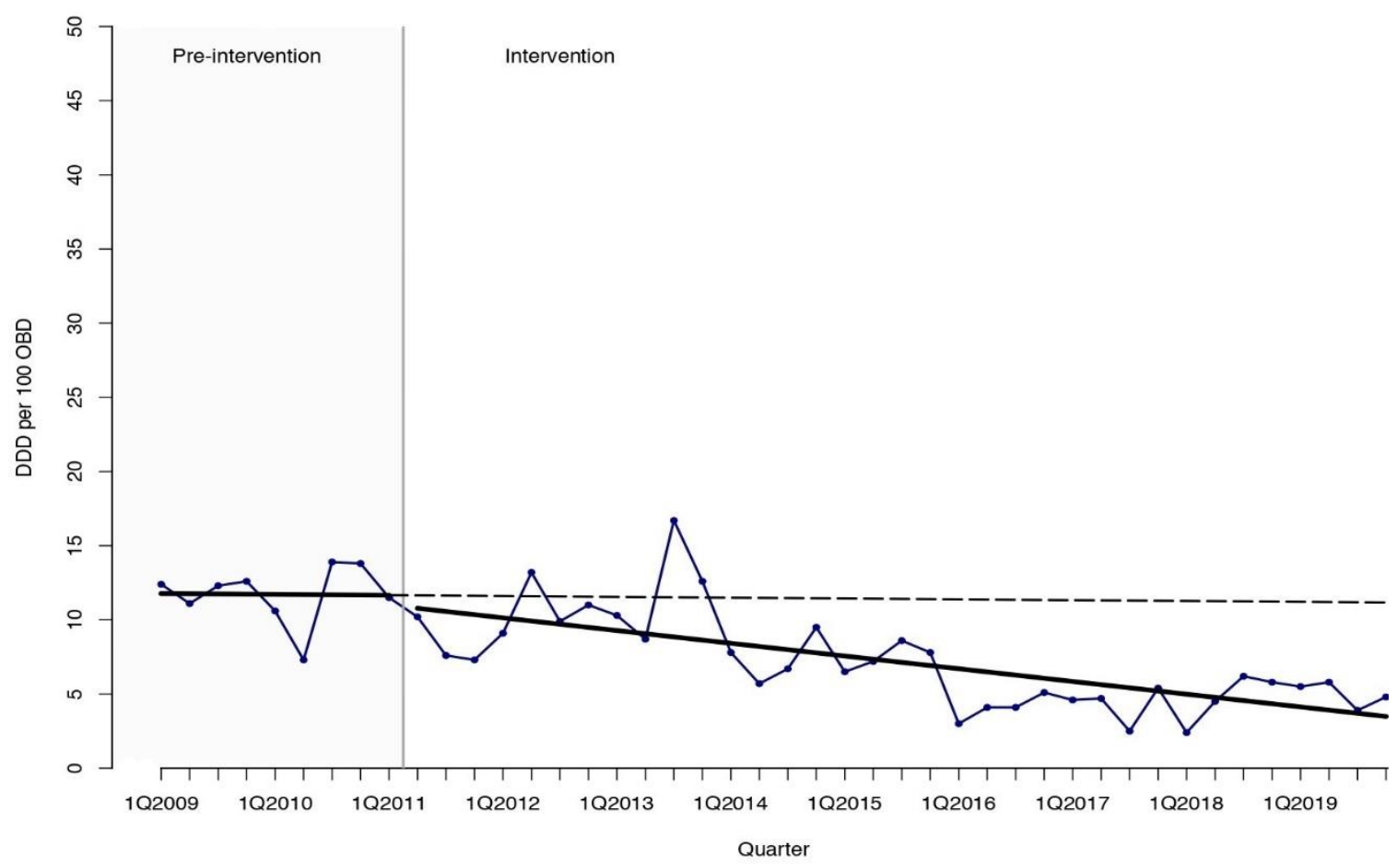

Quinolones

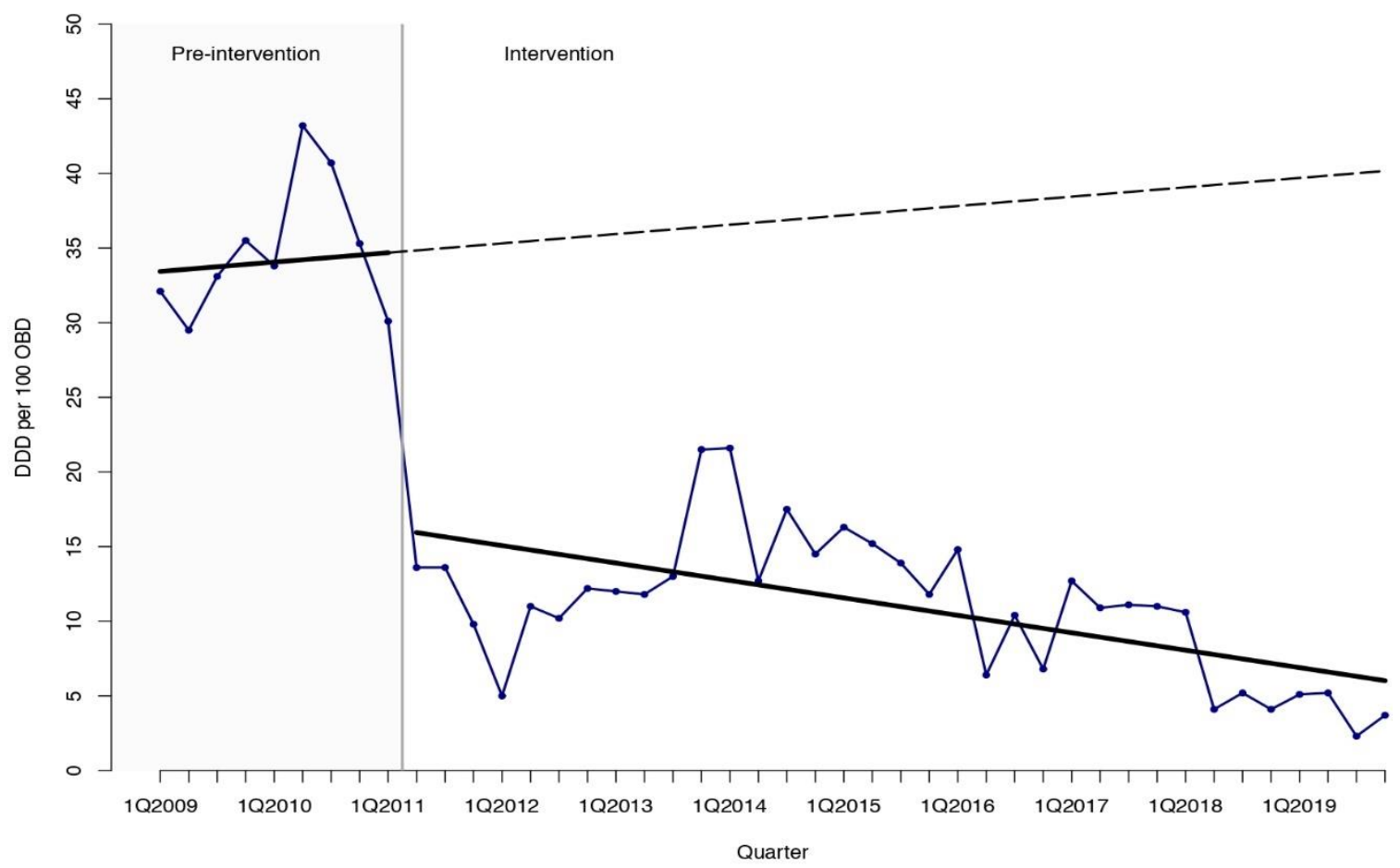

Figure 3. Interrupted time-series analysis of the trends in consumption for carbapenems and quinolones observed before and after the implementation of the antimicrobial stewardship program. Solid lines show the observed trend during the pre-intervention and intervention periods. Dashed lines show the expected trend after the intervention according to the pre-intervention values. DDD, defined daily doses. OBD, occupied bed days. Q, quarter. 


\subsection{Clinical Outcomes}

For the entire study period, the most common gram-negative microorganism causing BSI was non-extended-spectrum beta-lactamase (ESBL) producing Escherichia coli (48.1\%). MDR gram-negative bacteria and Candida spp. caused $14.4 \%$ and $5.6 \%$ of BSI that were monitored, respectively (Table S3). BSI produced by MDR Pseudomonas aeruginosa and Candida spp. were responsible for the highest values of early and late mortality rates (Table S4).

For incidence density (ID) and mortality rate, the ITS analysis is shown in Table 2. The pre-post analysis and the trend analysis can be found in Tables S5 and S6, respectively.

Table 2. Interrupted time-series analysis of changes in trends of incidence and mortality rate of multidrug-resistant bloodstream infections.

\begin{tabular}{|c|c|c|c|c|}
\hline Outcomes & $\begin{array}{c}\text { Pre-Intervention } \\
\text { Trend }\end{array}$ & $\begin{array}{c}\text { Change } \\
\text { in Level }^{\text {a }}\end{array}$ & $\begin{array}{c}\text { Change } \\
\text { in Trend }\end{array}$ & $\begin{array}{c}\text { Relative } \\
\text { Effect }^{\text {c } \%}\end{array}$ \\
\hline $\begin{array}{l}\text { Incidence } \\
\text { density }\end{array}$ & $\begin{array}{c}-0.09 \\
(-0.25 \text { to } 0.07)\end{array}$ & $\begin{array}{c}-0.11 \\
(-1.00 \text { to } 0.77)\end{array}$ & $\begin{array}{c}0.10 \\
(-0.06 \text { to } 0.26)\end{array}$ & $\begin{array}{c}98.9 \\
(-301.4 \text { to } 499.2)\end{array}$ \\
\hline Early mortality & $\begin{array}{c}0.009 \\
(-0.03 \text { to } 0.05)\end{array}$ & $\begin{array}{c}0.06 \\
(-0.14 \text { to } 0.26)\end{array}$ & $\begin{array}{c}-0.01 \\
(-0.05 \text { to } 0.03)\end{array}$ & $\begin{array}{c}-72.1 \\
(-147.8 \text { to } 3.5)\end{array}$ \\
\hline Late mortality & $\begin{array}{c}0.01 \\
(-0.06 \text { to } 0.08)\end{array}$ & $\begin{array}{c}-0.03 \\
(-0.42 \text { to } 0.36)\end{array}$ & $\begin{array}{c}-0.005 \\
(-0.08 \text { to } 0.07)\end{array}$ & $\begin{array}{c}-35.55 \\
(-346.9 \text { to } 275.8)\end{array}$ \\
\hline
\end{tabular}

Data are presented as quarterly incidence density and all-cause crude death rate per 1000 occupied bed days with a $95 \%$ confidence interval, unless otherwise specified. a Increase or decrease in the first quarter after the start of the antimicrobial stewardship program (ASP) period with respect to the expected value. ${ }^{b}$ Change in slope for the ASP period. ${ }^{c}$ Percentage difference between the expected value according to the pre-intervention trend and the trend nine years after the start of the ASP.

The ID of BSI caused by MDR organisms, which kept low during the entire study period, remained stable (mean incidence 1.11 episodes per 1000 OBD for the pre-intervention period and 0.82 episodes per 1000 OBD for the ASP period) with a quarterly percentage change (QPC) of $-0.3 \%$ (95\% CI -2.0 to $1.4, p=0.709)$. Early and late mortality of MDR BSI presented a steady trend with a QPC of $-0.7 \%(95 \% \mathrm{CI}-1.7$ to $0.3, p=0.154)$ and $-0.6 \%$ ( $95 \% \mathrm{CI}-1.5$ to $0.3, p=0.201)$, respectively.

\subsection{Changes in Healthcare during the Study Period}

Activity indicators related to the volume and complexity of the hematology department such as the number of blood cultures per 1000 OBD, total admissions, OBD, and the number of allogeneic hematopoietic stem-cell transplantation (HSCT) increased during the study period. Other indicators of the department's activity remained stable (Table 3). 
Table 3. Indicators related to the volume and complexity of the activity at the hematology department.

\begin{tabular}{|c|c|c|c|c|c|c|c|c|c|c|c|c|}
\hline Outcomes & 2009 & 2010 & 2011 & 2012 & 2013 & 2014 & 2015 & 2016 & 2017 & 2018 & 2019 & $\begin{array}{c}\text { APC } \\
(95 \% \mathrm{CI})\end{array}$ \\
\hline $\begin{array}{l}\text { Blood cultures } \\
\text { per } 1000 \text { OBD }\end{array}$ & 72 & 71 & 59 & 71 & 100 & 121 & 92 & 100 & 100 & 133 & 102 & $\begin{array}{c}6.014 \\
(2.348 \text { to } 9.811)\end{array}$ \\
\hline AML & 21 & 33 & 35 & 47 & 35 & 37 & 35 & 29 & 43 & 35 & 37 & $\begin{array}{c}4.400 \\
(-6.186 \text { to } 16.279)\end{array}$ \\
\hline Admissions & 1005 & 1055 & 1081 & 946 & 1052 & 1148 & 1169 & 1120 & 1133 & 1290 & 1265 & $\begin{array}{c}2.336 \\
(1.253 \text { to } 3.430)\end{array}$ \\
\hline OBD & 8966 & 9128 & 10,616 & 10,463 & 10,343 & 10,620 & 10,840 & 11,135 & 11,753 & 11,719 & 14,463 & $\begin{array}{c}3.540 \\
(2.358 \text { to } 4.735) \\
\end{array}$ \\
\hline $\begin{array}{l}\text { Length of stay, } \\
\text { mean }\end{array}$ & 16 & 15 & 18 & 17 & 17 & 16 & 16 & 16 & 17 & 14 & 16 & $\begin{array}{c}-0.843 \\
(-1.908 \text { to } 0.235)\end{array}$ \\
\hline $\begin{array}{c}\text { Allogeneic } \\
\text { HSCT }\end{array}$ & 19 & 20 & 33 & 40 & 47 & 55 & 56 & 58 & 44 & 43 & 47 & $\begin{array}{c}8.609 \\
(4.436 \text { to } 12.948)\end{array}$ \\
\hline $\begin{array}{c}\text { HSCT - related } \\
\text { mortality, } \%\end{array}$ & 5.3 & 5.0 & 0 & 0 & 6.4 & 1.8 & 7.1 & 1.7 & 0 & 2.3 & 2.1 & $\begin{array}{c}-7.007 \\
(-16.347 \text { to } 3.376)\end{array}$ \\
\hline
\end{tabular}

For each year, data are presented as the number of events, unless otherwise specified. In the last column, the annual percentage of change (APC) obtained from joinpoint regression analysis with a 95\% confidence interval (CI) is included. OBD, occupied bed days. AML, acute myeloid leukemia. HSCT-related mortality, hematopoietic stem-cell transplantation (HSCT)-related mortality within the first 100 days after allogeneic HSCT from human leukocyte antigen (HLA)-identical siblings.

\section{Discussion}

The results of our study show that an education-based ASP in the hematology department was able to achieve long-term reductions in overall antimicrobial consumption and improvements in the prescription profile, especially relevant in broad-spectrum antibiotics such as carbapenems, quinolones, and antifungals, without increasing the mortality rates and maintaining a low incidence of MDR BSI. This positive impact was observed in a tertiary care hospital where infectious diseases consultation (IDC) was performed for more than 25 years and up to the PRIOAM implementation. To the best of our knowledge, this is the first study proving nine years' data on the benefits of ASPs in the setting of hematological patients.

Very few previous studies have evaluated the effect of ASPs on antimicrobial consumption in hematological patients with most of them limited by sample size, study period ( $<2$ years before or after intervention), and the absence of data about specific groups of antibiotics. One of the most rigorous is the study performed by So et al. [10] in leukemia units with audit and feedback as the core measures of the ASP. In contrast to our results, the intervention was associated with a significant decrease in antibiotic use ( -35.1 DDD per 100 patient-days), but no significant trend in antifungal prescription was observed during a two-year period (-4.0 DDD per 100 patient-days). Two other research works with a one-year evaluation time and including solid and hematological malignancy patients showed favorable results after the beginning of antimicrobial stewardship strategies in terms of global antibiotic consumption [11] and meropenem prescription [7].

The increase of infections caused by MDR bacteria is a major health problem worldwide [12]. This challenge also affects hematological patients [13-15]. However, the percentage of MDR bacteria in our center was lower than previously reported by others [15-18], and notably, remained stable throughout the study period. The low prevalence of ESBL producing E. coli and MDR P. aeruginosa and, particularly, the absence of carbapenemaseproducing Enterobacteriaceae was especially important. The sustained reduction of the use of all-class antibiotics associated with the intervention has likely contributed to preventing the generalized increase in MDR infections described in other centers.

In our study, the early and late mortality rates from MDR BSI remained stable during the intervention, showing the absence of deleterious effects for reducing antimicrobial use in these patients. Death rates were higher for BSI caused by MDR organisms as 
described before [12,18]. Late mortality for ESBL producing Enterobacteriaceae was inferior to the 21-day mortality rate reported by Trecarichi et al. [18] for non-susceptible strains (26.2\%) and comparable for MDR P. aeruginosa (42.4\%). Despite the differences in study design, population and antimicrobial utilization due to different local treatment protocols and colonization rates by MDR bacteria, preceding results, similar to ours, illustrated the potential benefits of antimicrobial stewardship approaches. The adherence to ASP recommendation has demonstrated to be an effective and safe strategy with a $64 \%$ relative risk reduction in 28-day mortality [19] and a significant decrease in the fatality rate (from $30 \%$ to $11 \%$ ) [5] both in patients with febrile neutropenia and hematological or solid tumors. In patients with hematological diseases and HSCT recipients, stopping antimicrobial therapy early did not significantly increase the incidence of fever relapse and positive blood cultures or the mortality rate, with the advantage of the reduction in the use of antibiotics [20-22].

The results of the current study are even more remarkable if it is taken into consideration that most indicators related to the volume and complexity of the activity at the hematology department increased considerably during the study period. A fact that, in general, is related to a higher frequency of infectious complications and, as a consequence, higher consumption of antibiotics. Only the number of patients diagnosed with acute myeloid leukemia (AML), the length of hospital stay, and the transplant-related mortality within the first 100 days after allogeneic HSCT from human leukocyte antigen (HLA)identical siblings remained stable. The monitoring of changes in healthcare as an internal control as well as the largest period of study, spanning 11 years in total, are some of the strengths of this work. Additionally, the employment of ITS analyses, the preferential method to assess the impact of health interventions over time [23], and the consistent results throughout the variables evaluated, support a potential causality relation between the ASP implementation and the progressive reduction in the antimicrobial pressure. The stable trend in the mortality by MDR BSI supports the safety of the intervention.

PRIOAM's methods diverge from those ASP performed previously in patients with onco-hematological diseases in which educational initiatives were not incorporated as the core element of the program [11], or they were based on a sole recommendation (deescalation, discontinuation, antibiotic cycling, etc.) $[6,20,21]$ and/or a specific diagnosis [10], commonly febrile neutropenia [17,19-21]. The educational nature combined with real-time intervention(s) and the inclusion of patients with all types of hematological diseases comprise a differentiating feature of this work.

In patients with hematological diseases, post-chemotherapy febrile neutropenia was one of the most frequent infectious syndromes requiring antimicrobial courses. In this sense, the contribution of the results of the How Long clinical trial [24], led by investigators from our institution, to change the clinical practice and to decrease antibiotic overpressure in hematological patients was considerable. According to the main findings, in high-risk patients with hematological malignancies and febrile neutropenia, empirical antimicrobial therapy can be safely discontinued after $72 \mathrm{~h}$ of apyrexia and clinical recovery irrespective of their neutrophil count. It reinforced the previously published recommendation from the 4th European Conference on Infections in Leukaemia (ECIL-4) about empirical treatment of febrile neutropenia [25].

In our center, quinolones were agents commonly selected as an empirical combination therapy in patients with febrile neutropenia, especially in those with a suspected respiratory infection. Quinolones showed the highest reduction after the start of the intervention and the greatest decrease in the relative effect at the end of the study period. It could be explained by the fact that the ASP guidelines highly recommended the withdrawal of combination therapy $48 \mathrm{~h}$ after the start if an infection was not confirmed, or if it was presented and narrower spectrum antibiotics could be employed instead of quinolones. The implementation of this recommendation through the ASP has likely contributed to achieving this result. 
However, for this study, some limitations should be noted. First, the study design is not exempt from the possibility of ecologic bias, and, consequently, we could not unequivocally associate the results of incidence and mortality of MDR organisms to the ASP implementation. Although the volume and complexity of the activity in the hematology department were monitored, other potential confounding factors such as those related to patients and the center could possibly interfere with the outcomes. In addition, the single-center design limits the external validity of our results and makes it necessary to confirm the reproducibility of the findings in different settings. Second, the close relationship between the IDC in the pre-intervention period and the ASP made it difficult to elucidate the precise weight of each one on the outcomes achieved. Nonetheless, regarding the use of antimicrobials, the stable trends during the pre-intervention period suggest that the implementation of the ASP was necessary to achieve the goals. The sole IDC was insufficient to promote a change in the entire department, as reported in previous studies [26]. Finally, non-MDR BSI, invasive candidiasis (other than candidemia) and aspergillosis have not been examined in this study. The decreasing trend in overall antimicrobial consumption, including voriconazole and liposomal amphotericin B as common treatments for invasive infections caused by molds [27], suggests, at least, a steady frequency of these infections.

\section{Materials and Methods}

\subsection{Study Design and Period}

A quasi-experimental before-after study of ITS was performed. The PRIOAM implementation started in January 2011, and, since then, data were prospectively registered for a nine-year period. For the ITS analyses, the study period spanned 44 quarters (11 years) from January 2009 to December 2019.

\subsection{Setting}

The program was performed at the 39-bed hematology department of the University Hospital Virgen del Rocio (Seville, Spain), which is a teaching hospital providing a tertiarycare service in Southwest Spain. The hospital, with 1177 beds and 72 intensive care unit beds, is a referral-center for solid-organ and HSCT. Adult patients (aged $\geq 18$ years) receiving treatment for hematological malignancies or undergoing HSCT are treated in this unit. Throughout the last nine years, the hematology department has admitted a mean number of 1134 adult patients per year and has performed a mean of 108 autologous and allogeneic HSCT per year in adults.

\subsection{Intervention}

The PRIOAM's methods and global outcomes have already been published $[8,9]$. In brief, it comprises a bundle of educational strategies performed by a multidisciplinary team including infectious diseases physicians, microbiologists, pharmacists, intensive care physicians, pediatricians, and preventivists. The core elements of PRIOAM are summarized in the Supplementary material (Figure S1).

Because most EI were performed when a potentially inappropriate prescription was detected (i.e., use of carbapenems or combination therapy for $>48 \mathrm{~h}$, antibiotic duration $>7$ days or targeted therapies), the main messages tackled in EI were: early identification and management of severe infections, interpretation of microbiologic results, de-escalation and sequential oral treatments whenever possible, diversification of antimicrobial prescriptions, and training in the optimal duration of antimicrobial courses. The form employed for EI is included as Figure S2. No other interventions concerning antimicrobial use (i.e., antimicrobial policies, restrictions, etc.) were performed during the study period. The infection control program in the hematology ward consisted of the isolation in high-efficiency particulate air (HEPA) filters conditioned rooms of neutropenic and HSCT patients, and contact isolation of patients with MDR bacteria or respiratory viruses recovered from clinical samples. Local guidelines for antifungal prophylaxis did not change substantially during the intervention period. No additional measures were implemented regarding 
infection prevention, and antibiotic prophylaxis was not recommended for hematological patients in our center since 2005.

Before the start of the PRIOAM, a stable IDC program was running at the hematology department, consisting of bedside advice for the management of complex infections, quick report of all BSI, the production and application of local guidelines, updated every two years, for the prevention, diagnosis, and management of infections, and surveillance and analyses of MDR outbreaks. The usual IDC led the implementation of antimicrobial stewardship tasks in the hematology department.

\subsection{Study Measures}

Antimicrobial use was evaluated through quarterly measures of the antibiotic consumption of the Anatomical Therapeutical Chemical (ATC) group J01 (antibacterials for systemic use) and antifungals ATC group J02. Data about antimicrobial consumption were automatically generated by the electronic prescribing system, which provided information about the units (capsules, injection vials, etc.) used by each department. Consumption was calculated as DDD per $100 \mathrm{OBD}$, according to the ATC Classification methodology and the 2019 World Health Organization DDD values [28]. Because no DDD was suggested for liposomal amphotericin B, we considered the $210 \mathrm{mg}$ dose as the unit.

For the study period, BSI caused by the most relevant microorganisms in patients with hematological diseases (E. coli, Klebsiella pneumoniae, P. aeruginosa, and Candida spp.) were registered. The effect of the intervention on the number of BSI produced by MDR microorganisms (ESBL-producing Enterobacteriaceae, MDR P. aeruginosa, and Candida spp.) was monitored quarterly and presented as ID per 1000 OBD. The German Society for Hygiene and Microbiology criteria [29] was taken into account for MDR categorization. The analysis of antibiotic susceptibility and resistance mechanisms was performed following the European Committee on Antimicrobial Susceptibility Testing (EUCAST) criteria [30,31]

The effect on the mortality rates was assessed as the all-cause crude death rate $[9,32]$ (deaths per 1000 OBD per quarter) on day +7 (early mortality) and +30 (late mortality) after the diagnosis of BSI. Patients dying in less than $24 \mathrm{~h}$ after blood sample collection were not considered for the mortality analysis, as previously proposed [26,33,34], for a better selection of patients benefitting from the intervention targeting an optimized use of antimicrobials.

To analyze the effect of changes in the hematology department during the 11-year study period, we monitored yearly indicators related to the volume and complexity of the activity at the department that may influence the antimicrobial use, such as the number of blood cultures per $1000 \mathrm{OBD}$, new patients diagnosed with AML, admissions, and OBD, as well as the mean length of stay. We also monitored the number of allogeneic HSCT and the transplantation-related mortality within the first 100 days after allogeneic HSCT from HLA-identical siblings.

Because presentation, dissemination, and introduction activities of PRIOAM took place in the different departments of the hospital from January to 31 March 2011, we considered 1 April as the beginning of the intervention period for the analysis.

\subsection{Statistical Analysis}

For descriptive aims, categorical variables were presented as frequency distribution and percentages, and continuous variables were presented as means \pm standard deviations (SD). The Student's $t$-test or the Mann-Whitney U test were employed for univariate pre-post analyses, after checking for normality using the Kolmogorov-Smirnov test.

To assess the effect of the ASP, an ITS analysis was performed to estimate changes in the level and trends before and after the inception of the program. We used a generalized least squares regression approach accounting for autocorrelation by autoregressive moving-average (ARMA) models. The final model selection for each variable was based on the Akaike Information Criterion with validation of the autocorrelation structures by likelihood ratio tests [35]. The long-term effect attributable to the ASP for each outcome 
was estimated by calculating the relative effect, as the percentage difference between the values of the expected pre-intervention trend and the modeled trend at the end of the study. Alternatively, a joinpoint regression analysis was conducted to explore the trends of the time-series [36], calculating the QPC during the 11-year study period by using the Joinpoint software modeling annual percentage change calculation to our log-transformed quarterly data with autocorrelated error models.

Confidence intervals or $p$-values $(p)$ were included to show statistical significance. Differences were considered statistically significant at $p<0.05$ (2-tailed tests). Statistical analyses were performed with IBM SPSS Statistics software v. 23.0, R software v. 3.5.2 and Joinpoint Regression Program v. 4.6.0.0.

\subsection{Ethics Approval}

The study was conducted in accordance with the Declaration of Helsinki, and the protocol was approved by the Ethics Committee of the University Hospital Virgen del Rocio (Project identification code: PI-0361-2010).

\section{Conclusions}

These results allow us to state that an education-based ASP contributed significantly to the decreasing trend in the use of antimicrobials and, possibly, to maintain the low incidence of MDR BSI despite the increase in the volume and complexity of the activity at the hematology department over the study period. Death rates of BSI caused by MDR organisms were stable, showing that these interventions are safe in this vulnerable population.

Supplementary Materials: The following are available online at https:/ / www.mdpi.com/2079-6 382/10/2/136/s1. Table S1: Differences between the pre-intervention period and the antimicrobial stewardship program period regarding pre-post analysis of antimicrobial consumption. Table S2: Trend analysis of antimicrobial consumption (2009-2019). Table S3: Frequency of most relevant gramnegative microorganisms and Candida spp. as causative agents of bloodstream infections (2009-2019). Table S4: Mortality of patients with the most relevant gram-negative microorganisms and Candida spp. causing bloodstream infections (2009-2019). Table S5: Differences between the pre-intervention period and the antimicrobial stewardship program period regarding pre-post analysis of incidence and mortality rate of multidrug-resistant bloodstream infections. Table S6: Trend analysis of the incidence and mortality rate of multidrug-resistant bloodstream infections (2009-2019). Figure S1: Description of the core elements of PRIOAM. Figure S2: Form for PRIOAM educational interviews.

Author Contributions: Conceptualization, M.A.-G. and J.M.C. Methodology, A.B.G.-G. and G.P. Formal analysis, A.B.G.-G. and G.P. Investigation, A.B.G.-G., M.A.-G., G.P., J.A.L., I.E., and M.V.G.-N. Writing—original draft preparation, A.B.G.-G. Writing—review and editing, M.A.-G., G.P., J.A.L., I.E., E.R.-A., J.G.-C., N.R.-T., M.I.M.-C., J.F.F.-G., J.L.R.-O., M.V.G.-N., J.M., J.-A.P.-S., and J.M.C. All authors have read and agreed to the published version of the manuscript.

Funding: This work was supported by public funding from the Regional Health Ministry of Andalucia (grant number PI-0361-2010), which did not participate in the development of the program or the analysis of its results.

Institutional Review Board Statement: The study was conducted according to the guidelines of the Declaration of Helsinki, and approved by the Ethics Committee of the University Hospital Virgen del Rocio (Project identification code: PI-0361-2010).

Informed Consent Statement: Taking into consideration the risks and potential harms involved in the research, the Ethics Committee approved the exemption of informed consent.

Data Availability Statement: The data presented in this study are available on request from the corresponding author. 
Acknowledgments: The whole hematology department of the University Hospital Virgen del Rocio, whose close commitment to the program made its results possible. We also acknowledge the invaluable contribution of all the PRIOAM professionals: physicians, clinical microbiologists, pharmacists, nurses, and other members of the hospital. We thank the hospital manager and medical director, and the Andalusian Health Service of the Regional Ministry of Health of Andalucia (Spain) for supporting the ASP.

Conflicts of Interest: J.M.C. has received honoraria as a speaker from Novartis, Astellas Pharma, Pfizer, MSD, Janssen Pharmaceuticals, and AstraZeneca, outside the submitted work. He has also received report grants from Instituto de Salud Carlos III, Spanish Government, co-financed by the European Development Regional Fund "A way to achieve Europe", during the conduct of the study. No potential conflict of interest was reported by all other authors.

\section{References}

1. Barlam, T.F.; Cosgrove, S.E.; Abbo, L.M.; MacDougall, C.; Schuetz, A.N.; Septimus, E.J.; Srinivasan, A.; Dellit, T.H.; Falck-Ytter, Y.T.; Fishman, N.O.; et al. Implementing an antibiotic stewardship program: Guidelines by the Infectious Diseases Society of America and the Society for Healthcare Epidemiology of America. Clin. Infect. Dis. 2016, 62, e51-e77. [CrossRef] [PubMed]

2. Alevizakos, M.; Gaitanidis, A.; Andreatos, N.; Arunachalam, K.; Flokas, M.E.; Mylonakis, E. Bloodstream infections due to extended-spectrum $\beta$-lactamase- producing Enterobacteriaceae among patients with malignancy: A systematic review and meta-analysis. Int. J. Antimicrob. Agents 2017, 50, 657-663. [CrossRef] [PubMed]

3. Das, I.; Nightingale, P.; Patel, M.; Jumaa, P. Epidemiology, clinical characteristics, and outcome of candidemia: Experience in a tertiary referral center in the UK. Int. J. Infect. Dis. 2011, 15, e759-e763. [CrossRef] [PubMed]

4. Taur, Y.; Jenq, R.R.; Perales, M.A.; Littmann, E.R.; Morjaria, S.; Ling, L.; No, D.; Gobourne, A.; Viale, A.; Dahi, P.B.; et al. The effects of intestinal tract bacterial diversity on mortality following allogeneic hematopoietic stem cell transplantation. Blood 2014, 124, 1174-1182. [CrossRef] [PubMed]

5. Madran, B.; Keske, S..; Tokça, G.; Dönmez, E.; Ferhanoğlu, B.; Çetiner, M.; Mandel, N.M.; Ergönül, Ö. Implementation of an antimicrobial stewardship program for patients with febrile neutropenia. Am. J. Infect. Control 2018, 46, 420-424. [CrossRef]

6. Webb, B.J.; Majers, J.; Healy, R.; Jones, P.B.; Butler, A.M.; Snow, G.; Forsyth, S.; Lopansri, B.K.; Ford, C.D.; Hoda, D. Antimicrobial Stewardship in a Hematological Malignancy Unit: Carbapenem Reduction and Decreased Vancomycin-Resistant Enterococcus Infection. Clin. Infect. Dis. 2020, 71, 960-967. [CrossRef]

7. Mardani, M.; Abolghasemi, S.; Shabani, S. Impact of an antimicrobial stewardship program in the antimicrobial-resistant and prevalence of clostridioides difficile infection and amount of antimicrobial consumed in cancer patients. BMC Res. Notes 2020, 13, 246. [CrossRef]

8. Cisneros, J.M.; Neth, O.; Gil-Navarro, M.V.; Lepe, J.A.; Jiménez-Parrilla, F.; Cordero, E.; Rodríguez-Hernández, M.J.; Amaya-Villar, R.; Cano, J.; Gutiérrez-Pizarraya, A.; et al. Global impact of an educational antimicrobial stewardship program on prescribing practice in a tertiary hospital centre. Clin. Microbiol. Infect. 2014, 20, 82-88. [CrossRef]

9. Molina, J.; Peñalva, G.; Gil-Navarro, M.V.; Praena, J.; Lepe, J.A.; Pérez-Moreno, M.A.; Ferrándiz, C.; Aldabó, T.; Aguilar, M.; Olbrich, P.; et al. Long-Term Impact of an educational antimicrobial stewardship program on hospital-acquired candidemia and multidrug-resistant bloodstream infections: A quasi-experimental study of interrupted time-series analysis. Clin. Infect. Dis. 2017, 65, 1992-1999. [CrossRef]

10. So, M.; Mamdani, M.M.; Morris, A.M.; Lau, T.T.Y.; Broady, R.; Deotare, U.; Grant, J.; Kim, D.; Schimmer, A.D.; Schuh, A.C.; et al. Effect of an antimicrobial stewardship programme on antimicrobial utilisation and costs in patients with leukaemia: A retrospective controlled study. Clin. Microbiol. Infect. 2018, 24, 882-888. [CrossRef]

11. Yeo, C.L.; Chan, D.S.; Earnest, A.; Wu, T.S.; Yeoh, S.F.; Lim, R.; Jureen, R.; Fisher, D.; Hsu, L.Y. Prospective audit and feedback on antibiotic prescription in an adult haematology-oncology unit in Singapore. Eur. J. Clin. Microbiol. Infect. Dis. 2012, 31, 583-590. [CrossRef] [PubMed]

12. WHO. Antimicrobial Resistance. 2020. Available online: https://www.who.int/news-room/fact-sheets/detail/antimicrobialresistance (accessed on 12 December 2020).

13. Gudiol, C.; Tubau, F.; Calatayud, L.; Garcia-Vidal, C.; Cisnal, M.; Sánchez-Ortega, I.; Duarte, R.; Calvo, M.; Carratalà, J. Bacteraemia due to multidrug-resistant Gram-negative bacilli in cancer patients: Risk factors, antibiotic therapy and outcomes. J. Antimicrob. Chemother. 2011, 66, 657-663. [CrossRef] [PubMed]

14. Montassier, E.; Batard, E.; Gastinne, T.; Potel, G.; de La Cochetière, M.F. Recent changes in bacteremia in patients with cancer: A systematic review of epidemiology and antibiotic resistance. Eur. J. Clin. Microbiol. Infect. Dis. 2013, 32, 841-850. [CrossRef]

15. Garcia-Vidal, C.; Cardozo-Espinola, C.; Puerta-Alcalde, P.; Marco, F.; Tellez, A.; Agüero, D.; Romero-Santana, F.; Díaz-Beyá, M.; Giné, E.; Morata, L.; et al. Risk factors for mortality in patients with acute leukemia and bloodstream infections in the era of multiresistance. PLoS ONE 2018, 13, e0199531. [CrossRef] [PubMed] 
16. Averbuch, D.; Tridello, G.; Hoek, J.; Mikulska, M.; Akan, H.; Yanez San Segundo, L.; Pabst, T.; Özçelik, T.; Klyasova, G.; Donnini, I.; et al. Antimicrobial Resistance in Gram-Negative Rods Causing Bacteremia in Hematopoietic Stem Cell Transplant Recipients: Intercontinental Prospective Study of the Infectious Diseases Working Party of the European Bone Marrow Transplantation Group. Clin. Infect. Dis. 2017, 65, 1819-1828. [CrossRef] [PubMed]

17. Martinez-Nadal, G.; Puerta-Alcalde, P.; Gudiol, C.; Cardozo, C.; Albasanz-Puig, A.; Marco, F.; Laporte-Amargós, J.; MorenoGarcía, E.; Domingo-Doménech, E.; Chumbita, M.; et al. Inappropriate Empirical Antibiotic Treatment in High-risk Neutropenic Patients With Bacteremia in the Era of Multidrug Resistance. Clin. Infect. Dis. 2020, 70, 1068-1074. [CrossRef] [PubMed]

18. Trecarichi, E.M.; Pagano, L.; Candoni, A.; Pastore, D.; Cattaneo, C.; Fanci, R.; Nosari, A.; Caira, M.; Spadea, A.; Busca, A.; et al. Current epidemiology and antimicrobial resistance data for bacterial bloodstream infections in patients with hematologic malignancies: An Italian multicentre prospective survey. Clin. Microbiol. Infect. 2015, 21, 337-343. [CrossRef] [PubMed]

19. Rosa, R.G.; Goldani, L.Z.; dos Santos, R.P. Association between adherence to an antimicrobial stewardship program and mortality among hospitalised cancer patients with febrile neutropaenia: A prospective cohort study. BMC Infect. Dis. 2014, 14, 286. [CrossRef]

20. La Martire, G.; Robin, C.; Oubaya, N.; Lepeule, R.; Beckerich, F.; Leclerc, M.; Barhoumi, W.; Toma, A.; Pautas, C.; Maury, S.; et al. De-escalation and discontinuation strategies in high-risk neutropenic patients: An interrupted time series analyses of antimicrobial consumption and impact on outcome. Eur. J. Clin. Microbiol. Infect. Dis. 2018, 37, 1931-1940. [CrossRef]

21. Petteys, M.M.; Kachur, E.; Pillinger, K.E.; He, J.; Copelan, E.A.; Shahid, Z. Antimicrobial de-escalation in adult haematopoietic cell transplantation recipients with febrile neutropenia of unknown origin. J. Oncol. Pharm. Pract. 2020, 26, 632-640. [CrossRef]

22. Snyder, M.; Pasikhova, Y.; Baluch, A. Early Antimicrobial De-escalation and Stewardship in Adult Haematopoietic Stem Cell Transplantation Recipients: Retrospective Review. Open Forum Infect. Dis. 2017, 4, ofx226. [CrossRef] [PubMed]

23. De Kraker, M.E.A.; Abbas, M.; Huttner, B.; Harbarth, S. Good epidemiological practice: A narrative review of appropriate scientific methods to evaluate the impact of antimicrobial stewardship interventions. Clin. Microbiol. Infect. 2017, 23, 819-825. [CrossRef] [PubMed]

24. Aguilar-Guisado, M.; Espigado, I.; Martín-Peña, A.; Gudiol, C.; Royo-Cebrecos, C.; Falantes, J.; Vázquez-López, L.; Montero, M.I.; Rosso-Fernández, C.; de la Luz Martino, M.; et al. Optimisation of empirical antimicrobial therapy in patients with haematological malignancies and febrile neutropenia (How Long study): An open-label, randomised, controlled phase 4 trial. Lancet Haematol. 2017, 4, e573-e583. [CrossRef]

25. Averbuch, D.; Orasch, C.; Cordonnier, C.; Livermore, D.M.; Mikulska, M.; Viscoli, C.; Gyssens, I.C.; Kern, W.V.; Klyasova, G.; Marchetti, O.; et al. European guidelines for empirical antibacterial therapy for febrile neutropenic patients in the era of growing resistance: Summary of the 2011 4th European Conference on Infections in Leukemia. Haematologica 2013, 98, 1826-1835. [CrossRef] [PubMed]

26. Molina, J.; Noguer, M.; Lepe, J.A.; Pérez-Moreno, M.A.; Aguilar-Guisado, M.; de la Vega, R.L.; Peñalva, G.; Crespo-Rivas, J.C.; Gil-Navarro, M.V.; Salvador, J.; et al. Clinical impact of an educational antimicrobial stewardship program associated with infectious diseases consultation targeting patients with cancer: Results of a 9-year quasi-experimental study with an interrupted time-series analysis. J. Infect. 2019, 79, 206-211. [CrossRef] [PubMed]

27. Tissot, F.; Agrawal, S.; Pagano, L.; Petrikkos, G.; Groll, A.H.; Skiada, A.; Lass-Flörl, C.; Calandra, T.; Viscoli, C.; Herbrecht, R. ECIL-6 guidelines for the treatment of invasive candidiasis, aspergillosis and mucormycosis in leukemia and hematopoietic stem cell transplant patients. Haematologica 2017, 102, 433-444. [CrossRef]

28. WHO. Collaborating Center for Drug Statistics Methodology. DDD Definition and General Considerations. 2019. Available online: https:/ / www.whocc.no/ddd/ (accessed on 12 December 2020).

29. Mattner, F.; Bange, F.C.; Meyer, E.; Seifert, H.; Wichelhaus, T.A.; Chaberny, I.F. Preventing the spread of multidrug-resistant gram-negative pathogens: Recommendations of an expert panel of the German Society for Hygiene and Microbiology. Dtsch. Arztebl. Int. 2012, 109, 39-45. [CrossRef]

30. The European Committee on Antimicrobial Susceptibility Testing. EUCAST Guideline for the Detection of Resistance Mechanisms and Specific Resistances of Clinical and/or Epidemiological Importance. Version 2.0. 2017. Available online: https:/ /www.eucast. org/resistance_mechanisms/ (accessed on 12 December 2020).

31. The European Committee on Antimicrobial Susceptibility Testing. Breakpoint Tables for Interpretation of MICs and Zone Diameters. Version 10.0. 2020. Available online: http:/ / www.eucast.org (accessed on 12 December 2020).

32. Porta, M. (Ed.) A Dictionary of Epidemiology, 6th ed.; Oxford University Press: New York, NY, USA, 2014.

33. López-Cortés, L.E.; Del Toro, M.D.; Gálvez-Acebal, J.; Bereciartua-Bastarrica, E.; Fariñas, M.C.; Sanz-Franco, M.; Natera, C.; Corzo, J.E.; Lomas, J.M.; Pasquau, J.; et al. Impact of an evidence-based bundle intervention in the quality-of-care management and outcome of Staphylococcus aureus bacteremia. Clin. Infect. Dis. 2013, 57, 1225-1233. [CrossRef]

34. Lee Rachael, A.; Joanna, Z.; Camins, B.C.; Griffin, R.L.; Martin Rodriguez, J.; McCarty, T.P.; Magadia, J.; Pappas, P.G. Impact of infectious disease consultation on clinical management and mortality in patients with candidemia. Clin. Infect. Dis. 2018, 68, 1585-1587. [CrossRef]

35. Penfold, R.B.; Zhang, F. Use of interrupted time series analysis in evaluating health care quality improvements. Acad. Pediatr. 2013, 13, S38-S44. [CrossRef]

36. Kim, H.; Fay, M.P.; Feuer, E.J.; Midthune, D.N. Permutation tests for joinpoint regression with applications to cancer rates. Stat. Med. 2000, 19, 335-351. [CrossRef] 